Check for updates

Cite this: J. Anal. At. Spectrom., 2018, 33,294

Received 23rd September 2017 Accepted 13th December 2017

DOI: 10.1039/c7ja00319f

rsc.li/jaas

\section{Compact diode-pumped Nd:YAG laser for remote analysis of low-alloy steels by laser-induced breakdown spectroscopy}

\author{
V. N. Lednev, (D) ${ }^{* a b}$ A. E. Dormidonov, ${ }^{c}$ P. A. Sdvizhenskii, ${ }^{b}$ M. Ya Grishin, ${ }^{\text {ad }}$ \\ A. N. Fedorov, ${ }^{a}$ A. D. Savvin, ${ }^{c}$ E. S. Safronova ${ }^{a}$ and S. M. Pershin ${ }^{a}$
}

A low weight diode-pumped Nd:YAG laser (400 g, 1064 nm, 5 ns, 130 mJ per pulse) was developed for a compact laser-induced breakdown spectroscopy (LIBS) system to be installed on a robotized arm. Fiber optics delivery vs. conventional LIBS were compared for $\mathrm{C}, \mathrm{Si}, \mathrm{Mn}$ and $\mathrm{Cr}$ analysis in low-alloy steels. Fiber optics transformed the multimode laser beam to a flat-top beam with an improved fluence profile stability, resulting in shallow and more reproducible craters. A fast imaging study revealed that plasma generated by fiber optic pulses was plane-shaped, more uniform and dissipated two-fold faster compared with the plasma induced by direct laser beam focusing. Greater peak fluence for conventional LIBS provided plasma with 20-100 times more intensive emission due to the greater ablated mass, higher temperature and electron density. Improved reproducibility of shot-to-shot measurements was observed for plasma induced by fiber optic pulses, due to more stable ablation. The analytical capabilities of LIBS were compared for fiber optics vs. conventional LIBS in terms of calibration curve linearity, limits of detection and the root mean square error of the cross-validation procedure. Limits of detection for $\mathrm{Si}$, $\mathrm{Cr}$ and $\mathrm{Mn}$ were always better for direct laser beam focusing; however, more importantly, the conventional LIBS system provided quantitative analysis for carbon in low-alloy steels $(0.025-0.5 \% \mathrm{wt})$ with acceptable detection limits (55 ppm) while fiber optic pulses produced too-low intensity plasma.

\section{Introduction}

The growth in new technologies and blooming of industrial automatization presents a challenge in analytical chemistry for the development of new instrumentation with on-site measurement and quantitative analysis capability. ${ }^{1-3}$ In addition, such systems are urgently required for applications where environmental conditions are dangerous/hazardous for analysts or where the objects of interest themselves are not safe (nuclear materials, situations with a high concentration of beryllium, etc.). Laser-induced breakdown spectroscopy (LIBS) is a powerful analytical technique for the rapid multielemental analysis of almost any sample in any environment. During the past decades, numerous LIBS systems have been developed for industrial applications such as corrosion monitoring on a nuclear plant facility, ${ }^{4}$ high efficiency recycling sorting systems, ${ }^{1,5}$ controlling of Zn-coating thickness ${ }^{6}$ and molten steel analysis. ${ }^{7,8}$

${ }^{a}$ Prokhorov General Physics Institute, Russian Academy of Science, Moscow, Russia. E-mail: vasilylednev@gmail.com

${ }^{b}$ National University of Science and Technology MISiS, Moscow, Russia ${ }^{c}$ Lomonosov Moscow State University, International Laser Center, Moscow, Russia

${ }^{d}$ Moscow Institute of Physics and Technology (State University), Dolgoprudny, Moscow Region, Russia
A laser is a core element of any LIBS system as its characteristics (wavelength, pulse duration, power density, repetition rate, etc.) strongly influence the plasma properties, and its emissivity defines LIBS analytical capabilities., ${ }^{\mathbf{1 , 9 - 1 1}}$ Different types of laser sources have been utilized, depending on the LIBS analysis goals (major components vs. additives analysis) and specific environment conditions (high ambient temperature, dusty or radioactive environment, etc.). The pulsed solid state Nd:YAG laser is a "workhorse" for most industrial LIBS applications due to its reliability, compactness, low price and robustness. Depending on the application needs and specific conditions for on-site analysis, laser pulses can be delivered to the target in two ways. First, the laser system (laser head, power supply etc.) can be installed on-site, utilizing direct laser beam focusing via a simple or complex telescopic system. ${ }^{\mathbf{1 , 1 2 - 1 4}}$ If lineof-sight analysis cannot be carried out, then laser pulses can be delivered by fiber optics to provide more flexible solutions for LIBS applications., ${ }^{\mathbf{1 , 3}}$

Fiber optics delivery systems strongly affect the output laserpulse characteristics. The first problem is the significant limitation in terms of maximum transferred pulse energy due to fiber damaging by powerful nanosecond pulses. ${ }^{3}$ Large diameter core fibers $(1000-1500 \mu \mathrm{m})$ can be used for transferring up to $80 \mathrm{~mJ}$ ns pulses, but the fiber flexibility is poor, and they are difficult to handle due to the increased stiffness. The second 
problem is the greater divergence of fiber optic beams, which is two orders larger (tenths of radians) compared with the input laser beam (few milliradians). Davies et al. ${ }^{15}$ constructed a LIBS system with $100 \mathrm{~m}$ fiber optic cables for nuclear facility inspection. The fiber cable transferred $15 \mathrm{~mJ}$ pulses $(1064 \mathrm{~nm}$, $10 \mathrm{~ns}$ ), and focusing optics was located only $25 \mathrm{~mm}$ from the target to achieve a detection limit of the order of a few hundred ppm. To the best of our knowledge, the largest lens-tosample distance equals $50 \mathrm{~mm}$ for the fiber optic sampling head developed by Cremers et al. ${ }^{16}$ for heavy elements in soils by the LIBS technique.

Our goal was to develop a LIBS system for in situ and realtime multielemental analysis of samples synthesized by additive technologies. Specifically, the co-axial laser cladding technique utilizes selective melting of powders for the production of composite materials, such as the wear-resistance coatings of nickel-chromium alloys reinforced with tungsten carbide particles. ${ }^{17}$ Unfortunately, quality reproducibility of such coatings is low, and thus the LIBS instrument is believed to be a key tool for improving quality in real-time and in situ control of elemental composition during coating synthesis. A stand-off LIBS system is not a suitable solution due to the complexity of the cladding head movements (almost any direction) resulting in partial hiding of the synthesized coating by the robot arm or the synthesized detail. The LIBS instrument should be installed on a fully automatic robotized arm which has strict limitations in terms of weight; specifically, the sampling part of the LIBS system should not exceed one kilogram by mass. The smallest accessible distance between the melting spot and the instrument head is $400 \mathrm{~mm}$ due to the possibility of damaging optics by the ejected melt droplets. Such a system can be constructed with the help of fiber optics for laser-pulse delivery and plasmaemission collection. For example, Palanco and Laserna ${ }^{\mathbf{1 8}}$ developed a fully automated LIBS system for express stainless steel analysis. They used fiber optics to deliver laser pulses to the target (lens-to-target distance was $30 \mathrm{~mm}$ ) and to transfer plasma emission to the spectrometer. However, fiber optic LIBS cannot provide quantitative analysis of light elements at a distance of $400 \mathrm{~mm}$.

Light elements (carbon, silicon) are the key components defining wear-resistant coating quality (nickel-chromium alloys reinforced with tungsten carbide); however, LIBS quantitative analysis is challenging for carbon. Nickel-chromium alloys and tungsten carbide powder materials typically contain iron (few\% wt) which is known to have spectral interference with strong carbon lines, so carbon lines in ultraviolet (UV) and deep UV have to be utilized. ${ }^{19}$ A high-temperature laser plasma provides favorable conditions for carbon and silicon analysis due to the higher excitation energy levels for such species. Consequently, high peak energy density is needed to provide analytically meaningful plasma; thus, laser pulses of rather high energy $(>50 \mathrm{~mJ})$ need to be transmitted. The other drawback of fiber optics is the larger beam divergence. Typically, two lens systems are used to transfer fiber endpoints to face the target surface. The first lens (collimating lens) should fit the fiber optic numerical apertures to effectively collect the beam energy. The second lens (focusing lens) should be of short focal length in order to obtain a small laser spot. If one wants to focus a multimode fiber optic beam at a $400 \mathrm{~mm}$ distance, then two lenses with a $400 \mathrm{~mm}$ focal length are needed to transfer the fiber endpoint image to the target surface with 1:1 magnification. Simple geometry calculations indicate that large-scale optics ( $250 \mathrm{~mm}$ diameter in our case) were needed, which is not practicable due to the high weight.

In other words, we were challenged to develop a lightweight LIBS sampling system which could be installed on the robotic arm for the real-time and in situ multielemental analysis of coatings synthesized by additive technology. Such a system must quantitatively analyze light elements from a distance of $400 \mathrm{~mm}$. A powerchip Nd:YAG laser is a perspective and most lightweight solution for a number of LIBS applications; ${ }^{20-23}$ however, such lasers provide a few hundred $\mu \mathrm{J}$ pulses, which is not enough in our case. Recently, powerful fiber lasers have been recognized as perspective tools for LIBS. ${ }^{24-27}$ These lasers have adequate beams that can be focused into small spots. However, such lasers produce laser pulses a few tenths of nanoseconds in length, which are limited to a few mJ pulse energy. A new generation of compact diode-pumped solid state (DPSS) lasers can be a solution to fulfill these strict criteria since such lasers combine high power pulses, low divergence and low weight. In the belief that such a compact laser can be an alternative to fiber optic delivery in different LIBS applications requiring flexibility, a lightweight compact DPSS Nd:YAG laser was designed. In this study, we compared the LIBS analytical capabilities of the developed compact DPSS laser and fiber optic delivery for light element analysis (carbon, silicon) in low-alloy steels.

\section{Experimental}

In order to fulfill the requirements for high-energy pulses and small weight of the LIBS instrument head, a compact DPSS pulsed Nd:YAG laser OG-MPN/100 (1064 nm, 5 ns, $130 \mathrm{~mJ}$ per pulse, $M^{2} \approx 90,10 \mathrm{~Hz}$ ) was produced (at Lomonosov Moscow State University). The developed laser was based on $5 \times 50 \mathrm{~mm}$ composite $\mathrm{Nd}^{3+}: \mathrm{Cr}^{4+}$ :YAG crystal, which combines active medium, optical resonator, and Q-switch control in the monolithic element to ensure the immunity of the laser to severe shock and vibration. The composite laser crystal was pumped by three laser diode stacks SLM-3 «Inject $\gg$ (summed power 4 $\mathrm{kW}$ ). Pumping geometry is designed in such a way that in the center of the laser crystal a stable spot of the inverted population is formed which does not depend on the laser diode stacks temperature. This engineering stunt allowed the stable laser to run without water cooling; thus, a compact $(40 \times 80$ $\mathrm{mm}$ ) and low weight (400 g) laser head was constructed (Fig. 1) providing high-energy (130 mJ) nanosecond laser pulses.

A schematic diagram for the LIBS setup with fiber optic delivery $v s$. compact laser installation is presented in Fig. 2. We used the same spectrometer and collecting optics, with the only difference being the way of delivering the laser energy to the target. The first scheme implies compact laser installation. In such a case, the laser beam was focused through a plano-convex quartz lens $(F=150 \mathrm{~mm})$ normally to the sample surface 


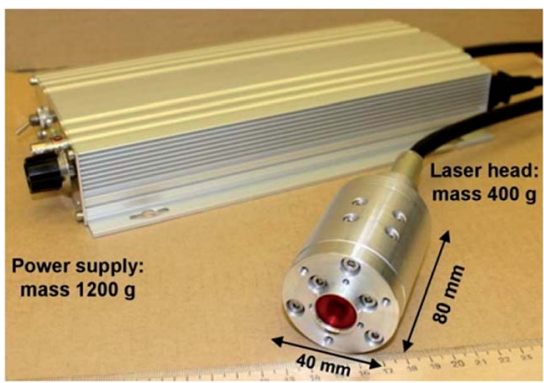

Fig. 1 Photo of compact DPSS Nd:YAG laser head (400 g) connected to a power supply $(1200 \mathrm{~g})$ via a $10 \mathrm{~m}$ cable.

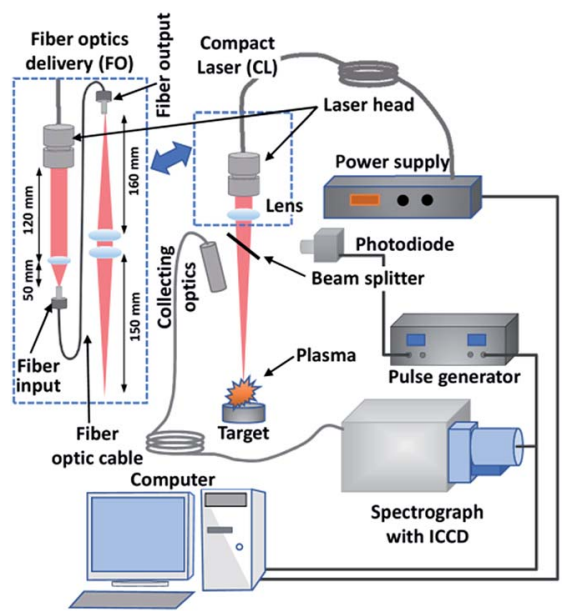

Fig. 2 Experiment setup scheme for comparison of compact laser (CL) vs. fiber optic (FO) delivery (laser beams are marked with red color).

located $148 \mathrm{~mm}$ from the lens. According to the second scheme, an $800 \mu \mathrm{m}$ quartz fiber $(1 \mathrm{~m}$ in length) was utilized to transport the laser radiation. The convenient way to deliver high-energy pulses through fiber optics is to couple a multimode optical fiber and multimode laser beam. Both the input and output fiber ends were cleaved and then cut at 90 degrees to obtain a perfect flat surface, which was controlled by optical microscopy. The laser crystal output endface image was decreased and projected by a $50 \mathrm{~mm}$ lens to the fiber input in order to achieve high efficiency of energy coupling and to prevent damage in the laser beam hot spots. The fiber entrance was placed slightly behind the lens focus; thus $80-90 \%$ of the core area was illuminated by the laser beam. To prevent the fiber optical breakdown, the laser-pulse energy was reduced to $26 \mathrm{~mJ}$. After fiber output, a two-quartz lens optical system was used (collimating lens $F=160 \mathrm{~mm}$; focusing lens $F=150 \mathrm{~mm}$ ) to focus the laser pulse at the sample surface. The overall efficiency of the laser energy transmittance through a $1 \mathrm{~m}$ long fiber and lens systems was estimated as $80 \%$.

In this study, we limited the lens-to-sample distance to $\sim 150 \mathrm{~mm}$ due to the low emissivity of the "fiber optics" plasma induced with longer focusing optics. Analytically meaningful laser plasma can be produced by direct focusing of laser pulses
(130 mJ) at distances up to $1000 \mathrm{~mm}$; however, we wanted to compare the LIBS analytical capabilities across two ways of delivering laser energy (fiber optics vs. compact laser head). Summing up, we have compared LIBS analysis with a fiber optic delivery system (20 mJ per pulse, FO20) and direct focusing of a laser beam with $130 \mathrm{~mJ}$ per pulse (CL130) and $20 \mathrm{~mJ}$ per pulse (CL20) energies. The last case was introduced to study the different laser beam profiles on LIBS analysis. A pair of neutral glass filters was used to decrease $130 \mathrm{~mJ}$ pulses (CL130) to $20 \mathrm{~mJ}$ (CL20). Plasma emission was collected by an UV-grade quartz lens $(F=150 \mathrm{~mm})$ and focused into a circular-to-rectangular optic-fiber bundle (13 quartz fibers with $100 \mu \mathrm{m}$ core diameters, length $10 \mathrm{~m}$ ) which was attached to the entrance slit (50 $\mu \mathrm{m}$ width, $4 \mathrm{~mm}$ high) of the spectrograph (Shamrock 303i, Andor) equipped with a gated charge-coupled device (CCD) camera (iStar DH180 Andor). Generally, passive Q-switch lasers provide a microsecond jitter of laser-pulse generation, so a gated CCD camera was externally synchronized by a PIN-photodiode. An additional pulse generator (DG535, SRS) was used to fit the photodiode pulse output voltage $(+9 \mathrm{~V})$ to the required TTL level $(<+5 \mathrm{~V})$ for the gated camera. The minimum synchronization delay for the pulse generator and camera electronics was estimated as $1 \mu \mathrm{s}$, so all plasma measurements were carried after this short delay. The sample was placed in a holder which was attached to a two-axis translation stage. In this study, we were interested in light element LIBS analysis (carbon, silicon) in low-alloy steels samples (Table 1). The sample surface was polished with sandpaper (ISO grit P2400) before measurements to improve ablation reproducibility.

\section{Results and discussion}

\subsection{Beam profiles in focal spot}

Laser beam fluence profiles at focal spots were compared for the compact laser (CL130) and fiber optic (FO20) delivery schemes (Fig. 3). Laser beam profiles were quantified by a CCD camera (DragonFly2, Point Grey Research) at different lens-to-sample distances. These measurements provided a quantitative estimation of the laser beam quality product $\left(M^{2}\right)$ and gave precise knowledge of the beam fluence $\left(\mathrm{J} \mathrm{cm}^{-2}\right)$ in the waist as well as its location relative to the sample surface. The laser beam quality $M^{2}$ was estimated according to the ISO standard. ${ }^{28} \mathrm{~A}$ quantitative beam quality comparison revealed a 30 -fold decrease of the $M^{2}$ product when fiber optics were used $\left(M^{2}=90\right.$ vs. $\left.M^{2}=3000\right)$. Fiber optics (FO20) transmission resulted in a flat-top beam profile with a $500 \mu \mathrm{m}$ diameter and $50 \mu \mathrm{m}$ wide wings. The beam profile and its pattern were highly reproducible for shot-to-shot measurements. In contrast to these results, direct beam focusing (CL130 and CL20) resulted in irregular shaped profiles in the focal spot. The CL130 beam had a $100 \mu \mathrm{m}$ wide core peak in its center and almost $500 \mu \mathrm{m}$ wide wings. The peak position and wing patterns slightly fluctuated for shot-toshot measurements, which is very typical for multimode lasing. Such difference in beam profiles provided three-fold higher peak fluence for the direct focusing scheme, even for the same pulse energy (CL20 vs. FO20). Note, that for all cases the laser beam fluence was far beyond the ablation threshold. Such 
Table 1 Reference low-alloy steel elemental composition, wt\%

\begin{tabular}{|c|c|c|c|c|c|c|c|c|c|c|c|c|c|c|}
\hline Sample & $\mathrm{C}$ & $\mathrm{Si}$ & $\mathrm{Mn}$ & $\mathrm{Cr}$ & $\mathrm{Ni}$ & $\mathrm{Cu}$ & $\mathrm{Al}$ & $\mathrm{Ti}$ & $\mathrm{V}$ & Mo & As & $\mathrm{Sn}$ & $\mathrm{Pb}$ & $\mathrm{Zn}$ \\
\hline Sample1 & 0.166 & 0.58 & 1.52 & 0.66 & 0.133 & 0.165 & 0.033 & 0.003 & 0.041 & 0.013 & 0.009 & 0.007 & 0.003 & 0.011 \\
\hline Sample2 & 0.328 & 0.67 & 0.96 & 0.038 & 0.060 & 0.059 & 0.005 & 0.0017 & 0.004 & 0.009 & 0.002 & - & - & - \\
\hline Sample3 & 0.348 & 1.25 & 0.91 & 1.16 & 0.133 & 0.76 & 0.015 & 0.004 & 0.006 & 0.011 & 0.005 & - & - & - \\
\hline Sample4 & 0.105 & 0.30 & 1.63 & 0.101 & 0.093 & 0.184 & 0.039 & 0.023 & 0.082 & 0.010 & 0.007 & 0.009 & 0.005 & 0.010 \\
\hline Sample5 & 0.0034 & 0.014 & 0.132 & 0.017 & 0.014 & 0.020 & 0.033 & 0.065 & 0.004 & 0.002 & 0.002 & 0.004 & - & - \\
\hline
\end{tabular}

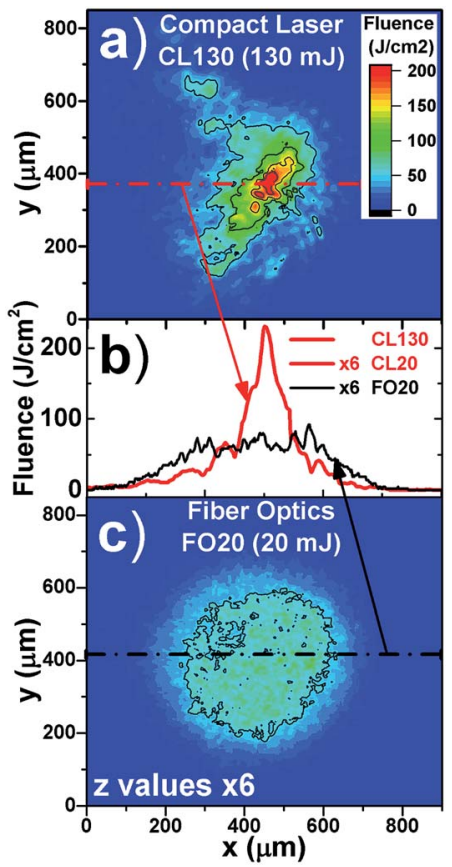

Fig. 3 Laser beam map profiles for compact laser (CL130) (a) and fiber optic (FO20) (c) in focal spot and corresponding cross-sections (b). A pair of neutral glass filters was used to decrease $130 \mathrm{~mJ}$ pulses (CL130) to $20 \mathrm{~mJ}$ (CL20), so the CL20 beam profile was the same as for CL130 but with six-fold lower fluence. Fiber optics maps and cross-section profiles were multiplied six-fold for a better view in the case of a FO20 beam.

a large difference in fluence profiles should strongly affect laser ablation, plasma properties and LIBS analysis for CL130, CL20 and FO20 sampling.

\subsection{Laser crater profiling}

Laser crater profiling was carried out to reveal the difference in ablated volume for CL130, CL20 and FO20 sampling. Laser craters produced by 100 pulses ablation were quantified by a white light interferometry microscope (NewView 6200, Zygo), and the results are presented in Fig. 4. A round-shaped shallow crater was obtained during ablation, with fiber-optic delivered pulses. The FO20 crater profile fit well to the corresponding beam profile. In the case of CL130 and CL20 ablation, the crater morphology was substantially different. The craters had irregular shapes and curvy pattern profiles at their bottoms. Similarities between the crater and beam profiles could be traced for CL20 ablation (Fig. 3a and 4b), while for CL130 sampling no matches were found. It should be noted that the FO20 crater profile was almost the same for replicate measurements, while CL130 and CL20 crater bottoms were different in parallel experiments. Interestingly, the estimated ablated volume for FO20 was two-fold smaller compared with the CL20 pulses,
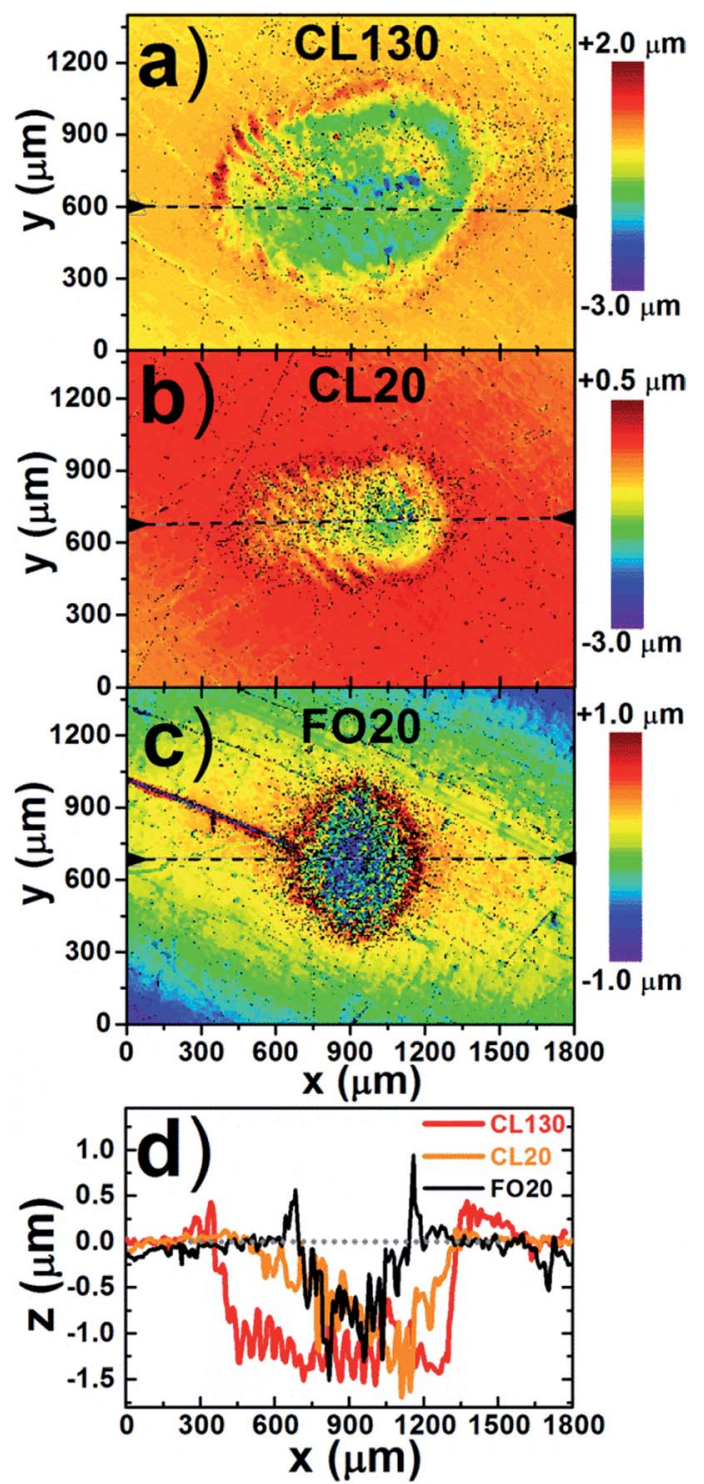

Fig. 4 Laser crater profiles for compact laser with pulse energy 130 $\mathrm{mJ}$ (CL130) (a) and $20 \mathrm{~mJ}$ (CL20) (b), and fiber optic delivery (FO20) (c) and corresponding cross-section comparison (d). The depth scales for $(a-c)$ profiles are different, thus (d) was plotted on the same scale. 
meaning that the ablation efficiency is better for a beam with a higher peak fluence. In the case of CL130 sampling, the laser crater volume increased four-fold compared with CL20. Consequently, the crater profiling study revealed that the fiber optic ablation resulted in a uniform crater profile, which is beneficial for applications requiring an accurate sampling strategy (depth profiling, cultural heritage studies). Direct beam focusing resulted in greater ablated mass; thus, it is convenient for applications focused on additives or/and light elements quantitative analysis.

\subsection{Plasma fast imaging}

The temporal evolution of the CL130, CL20 and FO20 plasma was studied by fast imaging (Fig. 5). Spectra integrated images $(350-850 \mathrm{~nm})$ of the laser plasma were projected by Canon objective (18-55 $\mathrm{mm} \mathrm{f/3.5-5.6)} \mathrm{and} \mathrm{quantified} \mathrm{by} \mathrm{the} \mathrm{gated} \mathrm{CCD}$ (iStar, Andor). The FO20 plasma had a plane-shape profile and was thermalized two-fold faster compared with CL20 plasma. CL20 ablation provided irregularly shaped plasmas which could be detected during the first $20 \mu \mathrm{s}$. Interestingly, CL130 plasma can be described as a plume with a "witches' hat" shape (cone shape with massive base) which changes its forms during the first $10 \mu \mathrm{s}$ and totally disappears within $50 \mu \mathrm{s}$. Such an unusual plasma shape was attributed to the CL130 fluence profile in the focal spot (Fig. 3): the central core plasma was induced by maximum peak fluence while ablation by beam "wings" produced weaker plasma.

\subsection{Plasma spectra}

The optimal choice of spectral region and analytical lines for LIBS depends on numerous factors including transition probabilities, spectral interference and the possibility of selfabsorption. Additionally, the chosen spectral regions should contain atomic/ionic lines of a matrix element in order to utilize an internal normalization procedure, which is a simple and effective way to improve precision for calibration curves. In this study we were focused on light element analysis (carbon, silicon); thus 190-205 and 275-290 nm spectral windows were chosen for spectra comparison. A strong Si I 288.16 line is widely used in LIBS for silicon analysis including low concentration samples. Deciding the optimal choice of analytical line for carbon analysis in low-alloy steels is a challenging task. ${ }^{19}$ For example, the strong carbon line C I 247.856 cannot be used for steel sample analysis due to spectral interference with the intense iron line Fe II 247.857. ${ }^{19,29}$ Carbon line C I 833.51 also interferes with very strong Fe I 833.19, Fe I 833.32 and Fe I 833.94 lines. ${ }^{30}$ Lines in the far-UV spectral range $(<190 \mathrm{~nm})$ are a better choice for carbon analysis due to the absence of spectral interference with iron lines; however these lines are strongly absorbed in air so evacuation or Ar atmosphere pumping is required. ${ }^{7}$ The atomic line C I 193.09 is a good choice for LIBS quantitative analysis due to minimal spectral interference with iron lines and moderate absorption by air and quartz optics. A list of analytical and reference lines used in this study is shown in Table 2.

Gated spectra were acquired for plasmas induced by CL130, CL20 and FO20 beams (Fig. 6). The spectra intensity in the 285$290 \mathrm{~nm}$ range was significantly lower for the FO20 case; however atomic/ionic lines ratios varied for CL130, CL20 and FO20 sampling. Gated spectra were summed to compare spectra which can be acquired with non-gated cameras. Timeintegrated intensity of strongest lines was 20-fold greater for plasma induced by direct beam focusing (CL20) rather than fiber optic delivery (FO20), while pulse energies were the same.

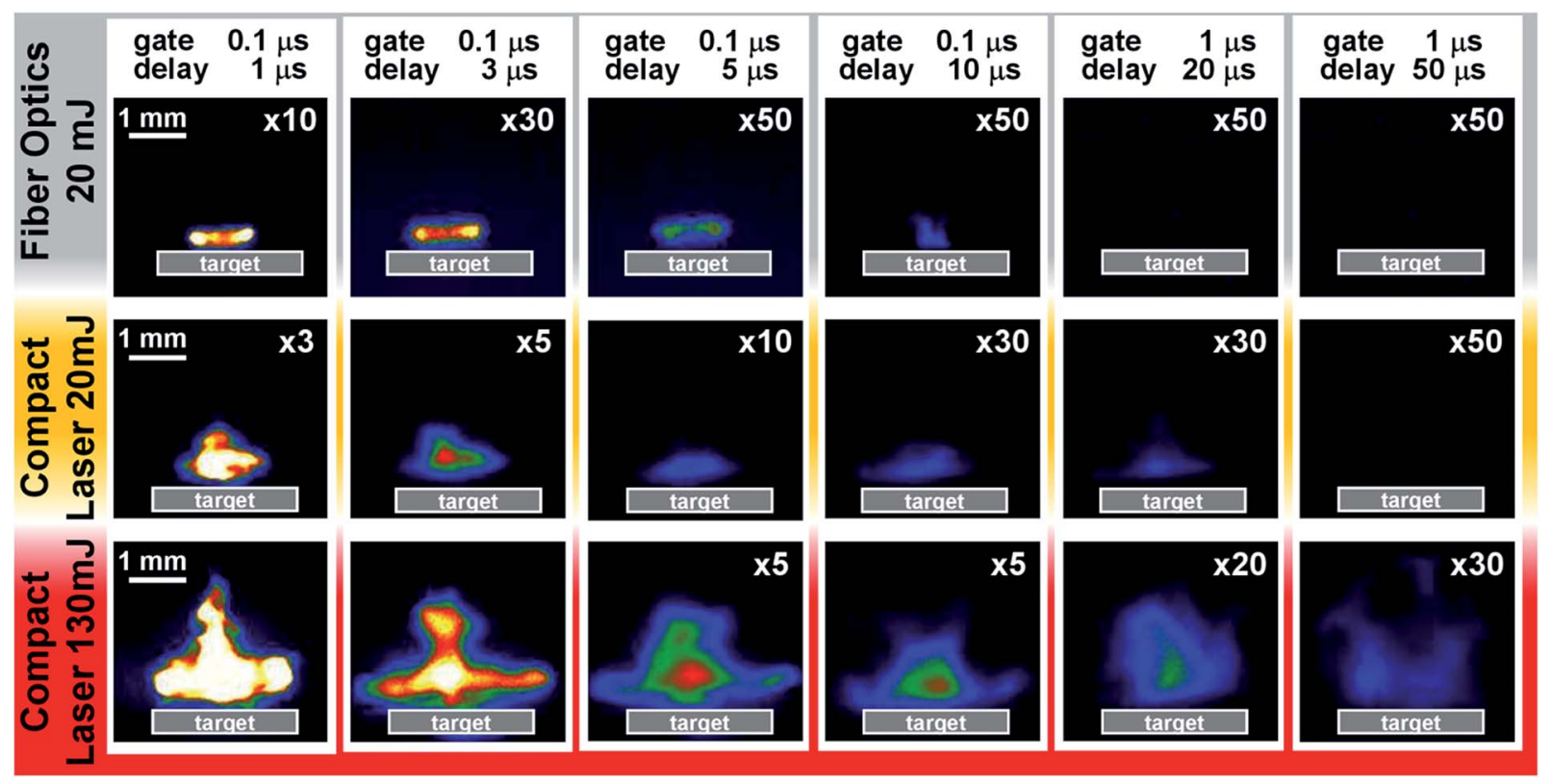

Fig. 5 Gated plasma imaging for plasma induced by laser pulses delivered with fiber optics (FO20) and compact laser with 20- (CL20) and 130 $\mathrm{mJ}$ (CL130) pulses. The intensity scale chart is the same for all images, but intensity was multiplied up to 50 times for a better view (see multiplier in the upper right corner of each image). 
Table 2 Atomic and ionic lines constants from National Institute of Standards and Technology (NIST) database: ${ }^{34}$ wavelength, transition probability, degeneracy of upper level, energy of upper level $\left(E_{k}\right)$ and energy of lower level $\left(E_{\mathrm{i}}\right)$. Analytical and matrix lines used for calibration are marked bold

\begin{tabular}{lllll}
\hline Wavelength, nm & $A_{\mathrm{ki}} \times 10^{7}, \mathrm{~m}^{-1}$ & $g_{\mathrm{k}}$ & $E_{\mathrm{i}}, \mathrm{eV}$ & $E_{\mathrm{k}}, \mathrm{eV}$ \\
\hline C I 193.09 & $\mathbf{3 3 . 9}$ & $\mathbf{3}$ & $\mathbf{1 . 2 6 4}$ & $\mathbf{7 . 6 8 5}$ \\
Fe II 204.07 & $\mathbf{4 . 6}$ & $\mathbf{1 0}$ & $\mathbf{1 . 9 6 4}$ & $\mathbf{8 . 0 3 8}$ \\
Cr II 283.56 & $\mathbf{2 0}$ & $\mathbf{1 2}$ & $\mathbf{1 . 5 4 9}$ & $\mathbf{5 . 9 2 0}$ \\
Si I 288.16 & $\mathbf{1 8 . 9}$ & $\mathbf{3}$ & $\mathbf{0 . 7 8 1}$ & $\mathbf{5 . 0 8 2}$ \\
Mn I 279.48 & $\mathbf{3 7 . 0}$ & $\mathbf{8}$ & $\mathbf{0 . 0}$ & $\mathbf{4 . 4 3 4}$ \\
Fe II 276.18 & $\mathbf{1 . 3 8}$ & $\mathbf{4}$ & $\mathbf{1 . 0 9 7}$ & $\mathbf{5 . 5 8 5}$ \\
Fe I 370.93 & 1.56 & 7 & 0.915 & 4.256 \\
Fe I 372.76 & 2.25 & 5 & 0.958 & 4.283 \\
Fe I 373.49 & 9.02 & 11 & 0.859 & 4.177 \\
Fe I 374.56 & 1.15 & 7 & 0.087 & 3.396 \\
Fe I 376.55 & 9.8 & 15 & 3.236 & 6.528
\end{tabular}

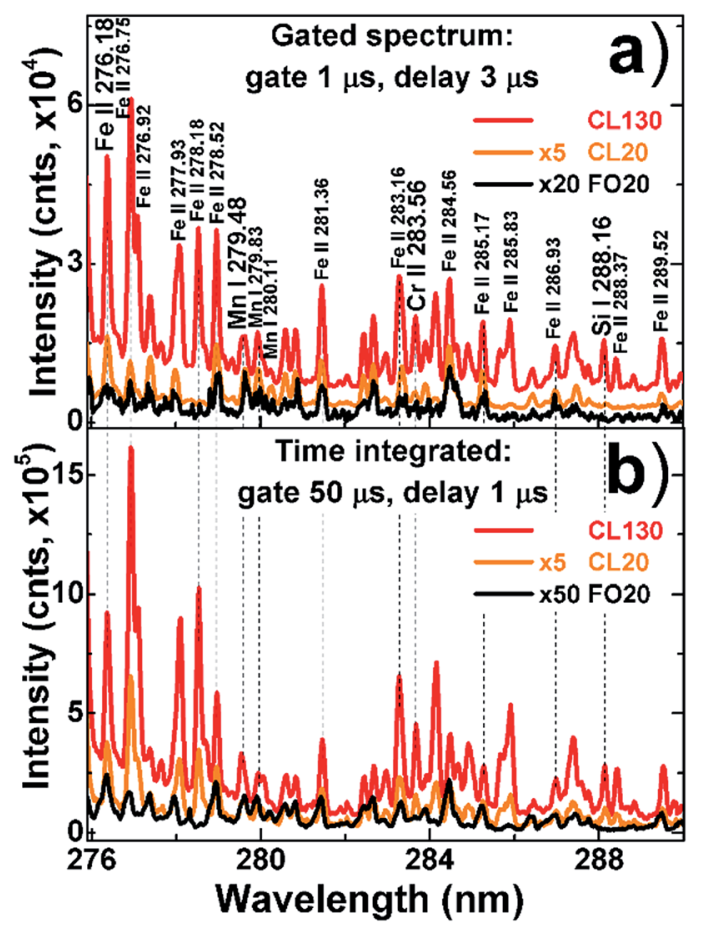

Fig. 6 Gated (a) and time-integrated (b) spectra for plasma induced by laser pulses delivered with fiber optics (FO20, black color) or direct laser beam focusing with $20 \mathrm{~mJ}$ (CL20, orange color) and $130 \mathrm{~mJ}$ (CL130, red color) energy pulses.

\subsection{Temperature and electron density}

Temperature and electron density are key parameters defining material atomization and atom/ion excitation in plasma. A systematic comparison of temperature and electron density temporal evolution was carried out for plasmas induced by CL130, CL20 and FO20 pulses. Temperature was estimated by the Boltzmann plot method with non-resonant iron lines (Table 2) as recommended by Aragón et al. ${ }^{31}$ Plasma electron density was measured by the Stark broadening of the Fe I 538.34 line. ${ }^{32}$ The line profile was fitted with Voigt function, and its full width at half maximum (FWHM) value was corrected on the spectrometer instrumental profile.

The results (Fig. 7) showed that the CL130 plasma had the highest temperature $(6500 \mathrm{~K})$, which slowly decreased during plasma expansion. If the same pulse energy was delivered to the sample surface, but the beam profiles were different (FO20 vs. CL20), then the fiber optic delivered laser pulses (FO20)induced cooler plasma. The electron density was higher for CL130 plasma, but its decay was also faster. FO20 and CL20 plasmas had nearly the same electron densities during the first microsecond, but FO20 plasma electron density dissipated more rapidly.

These results are consistent with previous studies for plasma induced by fiber optic excitation. ${ }^{25}$ Lower temperatures and electron densities and faster decays should be attributed to rapid cooling of plane-shape FO20 plasma.

To sum up, multimode fiber optic transmission resulted in a flat-top laser beam profile with improved stability. However, lower fluence for the beam profile produced a plane-shaped plasma which was rapidly thermalized due to a lower initial temperature and greater surface-to-plasma-mass ratio. Direct laser beam focusing (CL20) resulted in a cone-shaped plasma with high gradients of material density, temperature and electron density. Such a shape provided more favorable conditions for atoms/ions excitation in the plasma due to a smaller surfaceto-plasma-mass ratio. Energy from the "hot" plasma center transferred to the outer plasma layers due to multiple collisions between plasma particles (atoms, ions) so energy was effectively utilized for additional atom/ion excitation before it was lost for LIBS by collisions with air molecules. In other words, sphereshaped plasma is the most effective way to shield a "hot"

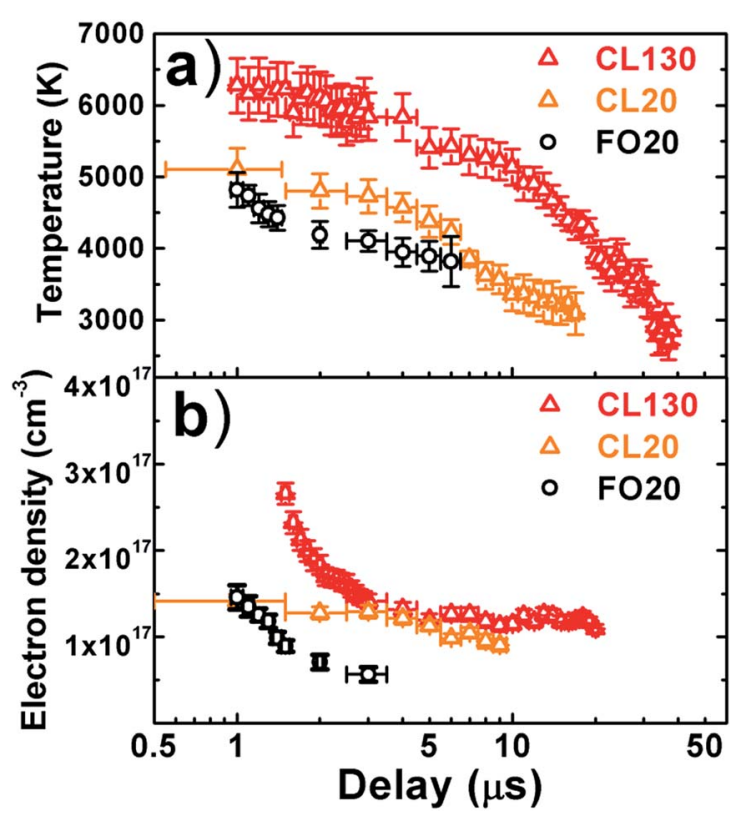

Fig. 7 Temperature (a) and electron density (b) evolution for laser plasmas produced by direct focusing of $130 \mathrm{~mJ}$ (CL130, red color) and $20 \mathrm{~mJ}$ (CL20, orange color) laser pulses, as well as fiber optic transmitted $20 \mathrm{~mJ}$ (FO20, black color) laser pulses. 
plasma center from collisions with air molecules, thus enhancing plasma-emission duration.

\subsection{Quantitative analysis}

In order to prevent the possible influence of sample surface contaminations and oxide layers on laser ablation, a few pretreatment pulses should be fired before acquiring the plasma spectrum. ${ }^{33}$ To optimize the number of "cleaning" pulses and compare LIBS signal reproducibility, we measured a few series of 300 successive single-shot spectra for plasmas induced by CL130, CL20 and FO20 pulses. Results of shot-to-shot reproducibility for the silicon line Si I 288.16 integral normalized on iron line (Fe II 276.18) integral are presented in Fig. 8. The best signal reproducibility (relative standard deviation) was observed for laser ablation with fiber optic pulses FO20. Slightly poorer reproducibility was observed for CL20 pulse, while for CL130 sampling, the signal variability doubled. Recently, we demonstrated that laser sampling with multimode laser beams results in self-induced reproducibility reduction due to fluctuations of the laser beam profile in the focal spot. ${ }^{9}$ Fiber optics improved the output fluence profile stability; thus, laser ablation steadiness was better. According to Fig. 8, different numbers of pretreatment pulses should be introduced before LIBS measurements. For example, FO20 sampling needed only a few pulses while CL20 and CL130 ablation required 20 and 50 cleaning pulses correspondingly to achieve stable ablation. In order to improve signal reproducibility, the LIBS spectrum from several laser shots has to be accumulated to reduce statistical errors. Calibration curves were constructed for every spectrum acquired by summing 100 single-shot spectra after the appropriate number of cleaning pulses.

Convenient gating is an important feature of LIBS measurements since better quality spectra can be acquired.

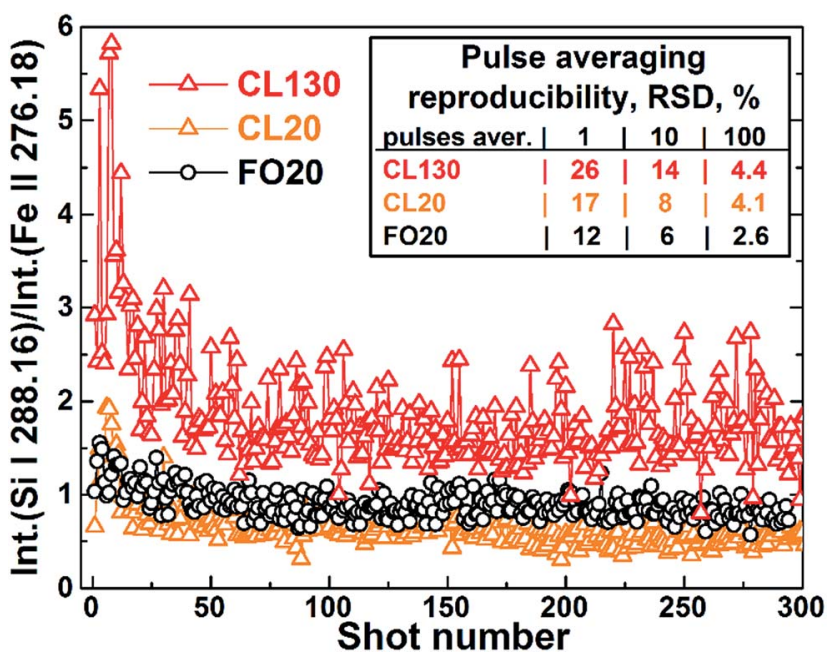

Fig. 8 Shot-to-shot signals ratios (Int.[Si I 288.16]/Int.[Fe 276.18] ratio) reproducibility for laser ablation by compact laser with direct focusing of 130- (CL130, red color) and $20 \mathrm{~mJ}$ pulses (CL20, orange color) as well as for fiber optic transmitted $20 \mathrm{~mJ}$ (FO20, black color) laser pulses. Relative standard deviation (RSD) was calculated for 1, 10 and 100 datasets.
Gating allows avoidance of intense continuum emission and acquisition of plasma spectra when atomic/ionic lines are not strongly broadened due to high electron density. This makes it possible to obtain spectra with a high intensity, low background and with minimal spectral interference. Here, we chose optimal gating parameters (gate width and delay) based on better signalto-noise ratios for analytical lines evolution: $1 \mu$ s gate with $1 \mu \mathrm{s}$ delay for 190-205 nm spectral range; $2 \mu$ s gate with 1- vs. $2 \mu \mathrm{s}$ delays for FO20 vs. CL20/CL130 plasmas in the 275-290 nm spectral range.

The experimental setup was calibrated for four elements (C, $\mathrm{Si}, \mathrm{Cr}, \mathrm{Mn})$. The internal normalization procedure, which utilizes an analyte to matrix line integral ratios instead of net integrals, was adopted to improve precision due to the shot-toshot fluctuation of LIBS signals. ${ }^{35}$ Atomic/ionic line integrals were background corrected. Our spectrometer system can acquire emission in the $18 \mathrm{~nm}$ spectral window in a single run, so choice of matrix lines for normalization was a compromise solution, still improving the calibration curves. Carbon line C I 193.09 was normalized on the Fe II 204.07 line, and all other analytical lines in the $275-290 \mathrm{~nm}$ region were rationed by the Fe II 276.18 line (Table 2). Five different locations at the target surface were probed to provide parallel measurements for every sample. Examples of calibration curves for chromium and silicon are presented in Fig. 9. The vertical error bars on the calibration curves show the standard deviations calculated from

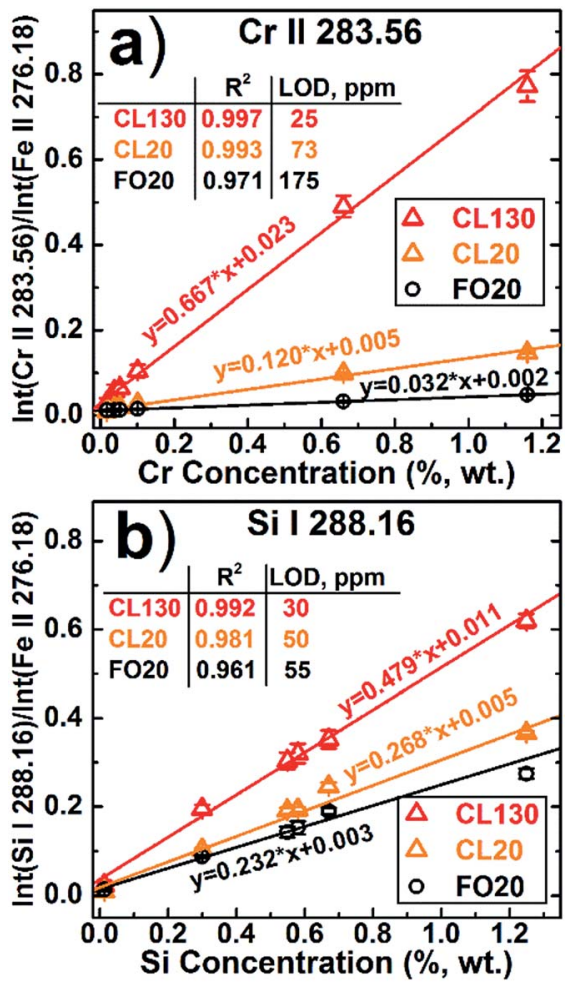

Fig. 9 Calibration curves for chromium (a) and silicon (b) for plasmas induced by fiber optic transmitted $20 \mathrm{~mJ}$ pulses (FO20, black color) as well as by direct focusing of 130 - (CL130, red color) and $20 \mathrm{~mJ}$ pulses (CL20, orange color). Spectra were acquired with $2 \mu$ sate and 1- and $2 \mu$ s delays for FO20 and CL20/CL130 plasma correspondingly. 
Table 3 Analytical capabilities comparison of $\mathrm{Cr}$, Si, Mn and C for CL130 vs. CL20 vs. FO20 LIBS: $R$-square $\left(R^{2}\right)$ factor, root mean square error RMSECV (wt\%), and LODs

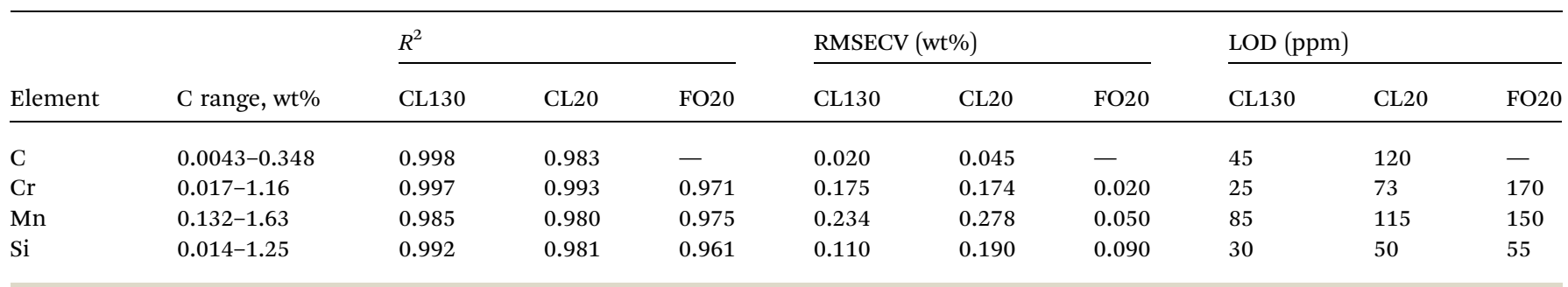

five replicate measurements. The obtained calibration curves were fitted with a linear function.

LIBS analytical capabilities for the different laser sources were compared in terms of precision, calibration curve linearity and limits of detection (LOD). In order to determine the LOD, ideally a number of reference samples with the lowest analyte concentration should be used. However, as the number of reference samples was limited, the LOD was extracted from calibration curves with $3 \sigma$ criteria, as recommended by IUPAC: LOD $=3 \sigma / s$, where $\sigma$ is a standard deviation of the background normalized on iron line signal for a sample with the lowest analyte content, $s$ is the calibration curve slope.

In order to evaluate detection accuracy, the leave-one-out cross-validation procedure was used with the root mean

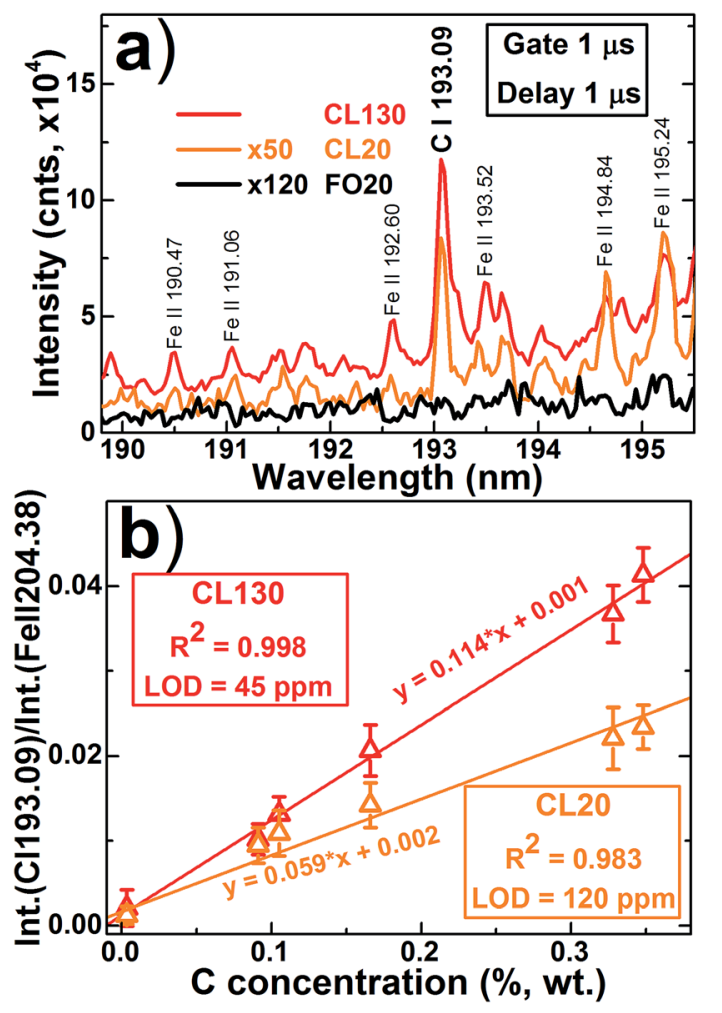

Fig. 10 Spectra in 190-195 nm range (a) and carbon (C I 193.09) calibration curves for plasma produced by compact laser with direct focusing of 130- (CL130, red color) and (CL20, orange color) and fiber optic transmitted $20 \mathrm{~mJ}$ (FO20, black color) pulses. Spectra (a) were acquired with $1 \mu$ s delay and $1 \mu$ s gate for the sample with $0.348 \%$ wt carbon concentration. square error of cross-validation (RMSECV) as the main index estimating the calibration model performance:

$$
\mathrm{RMSECV}=\sqrt{\frac{1}{n} \sum_{i}^{n}\left(c_{i}-c_{i}^{\mathrm{ref}}\right)^{2}},
$$

where $c_{i}$ is the predicted concentration, $c_{i}^{\text {ref }}$ is the reference concentration and $n$ is the number of calibration samples. Results on LIBS analytical capabilities comparison for the fiber optic delivery (FO20) and direct laser beam focusing (CL20, CL130) are summarized in Table 3.

Calibration curve linearity ( $R^{2}$ factor) was comparable or slightly better for CL130 LIBS. However, a small self-absorption was observed for silicon calibration curves in all cases for concentrations beyond $0.8 \mathrm{wt} \%$. For all elements of interest, better LOD results (30-85 ppm) were achieved for CL130 pulses due to brighter and longer plasma emission owing to greater pulse energy utilized for sampling. Fiber optic LIBS provided better precision and comparable linearity; thus, RMSECV values were always better than those for conventional LIBS. These results are consistent with previous studies on fiber optic LIBS. $^{25}$

Examples of LIBS spectra in the 190-195 $\mathrm{nm}$ range for plasma produced by CL130, CL20 and FO20 pulses are presented in Fig. 10. Plasma emission was strongly absorbed by $150 \mathrm{~cm}$ air and $10 \mathrm{~m}$ quartz optical path; however meaningful signal-to-noise ratio spectra can be acquired for CL130 and CL20 pulses. Laser plasma induced by FO20 pulses was too weak, and no C I 193.09 line was detected in the spectrum. Calibration curves were constructed for the carbon line C I 193.09 with normalization on the Fe II 204.38 line for CL130 and CL20 ablating pulses (Fig. 10). The higher intensity for the CL130 spectrum resulted in a better calibration curve linearity ( $R$-squares) and improved sensitivity, as well as in better RMSECV (Table 3). The estimated LOD for carbon detection (55 ppm) was improved compared with previous LIBS studies when ablation was made in air, ${ }^{36}$ but was poorer compared with farUV region carbon lines which require vacuum/argon conditions..$^{7,37,38}$

\section{Conclusions}

In summary, a new lightweight compact DPSS Nd:YAG laser (400 g, $1064 \mathrm{~nm}, 5 \mathrm{~ns}, 130 \mathrm{~mJ}$ per pulse) was developed for a compact LIBS system which can be installed on a robotized arm and will be capable analyzing low-alloy steel melts. Fiber 
optic delivery $v s$. conventional LIBS were compared for C, Si, Mn and $\mathrm{Cr}$ analysis in low-alloy steels. The fiber optics delivery scheme transformed the multimode laser beam to a flat-top beam with improved stability of the fluence profile in the focal spot. A flatter laser beam profile produced shallow and more reproducible craters for fiber optic LIBS. A rapid imaging study revealed that plasma generated by fiber optic pulses was weaker, more uniform, and dissipated two-fold faster compared with the conventional LIBS pulses with the same energy. Increased ablated mass and greater peak fluence for conventional LIBS setup provided plasma with 20-100 times more intensive emission compared with the fiber optic case. Temperature and electron density were higher and reduced more slowly for plasma induced by direct laser beam focusing. Multimode laser beam ablation produced cone-shaped plasma which provided more favorable conditions for atoms/ion excitation in the plasma due to more effective shielding of "hot" plasma center from collisions with air molecules; thus, enhancing plasma-emission duration.

An improved reproducibility of shot-to-shot measurements was observed for plasma induced by fiber optic pulses due to better reproducibility of laser beam profile resulting in more stable ablation. The calibration curve linearity was better for the conventional LIBS system. However, better RMSECVs were calculated for fiber optic LIBS due to improved reproducibility of laser ablation and more uniform plasma. Limits of detection for $\mathrm{Si}, \mathrm{Cr}$ and $\mathrm{Mn}$ were always better for conventional LIBS due to greater peak fluence in the focal spot, resulting in larger ablated volume and higher plasma temperature. More important is that the conventional LIBS system provided quantitative analysis for carbon in low-alloy steels (0.025-0.5\% wt) with acceptable detection limits (55 ppm), while laser pulses delivered by fiber optics produced weak plasma and low-quality spectra.

\section{Conflicts of interest}

There are no conflicts to declare.

\section{Acknowledgements}

The authors gratefully acknowledge the financial support of the Russian Science Foundation (agreement No. 16-19-10656).

\section{References}

1 R. Noll, H. Bette, A. Brysch, M. Kraushaar, I. Mönch, L. Peter and V. Sturm, Spectrochim. Acta, Part B, 2001, 56, 637-649.

2 G. Galbács, Anal. Bioanal. Chem., 2015, 407, 7537-7562.

3 J. F. Fortes and J. J. Laserna, Spectrochim. Acta, Part B, 2010, 65, 975-990.

4 A. I. Whitehouse, J. Young, I. M. Botheroyd, S. Lawson, C. P. Evans and J. Wright, Spectrochim. Acta, Part B, 2001, 56, 821-830.

5 R. Noll, I. Mönch, O. Klein and A. Lamott, Spectrochim. Acta, Part B, 2005, 60, 1070-1075.
6 H. Balzer, S. Hölters, V. Sturm and R. Noll, Anal. Bioanal. Chem., 2006, 385, 234-239.

7 L. Peter, V. Sturm and R. Noll, Appl. Opt., 2003, 42, 61996204.

8 G. Hubmer, R. Kitzberger and K. Mörwald, Anal. Bioanal. Chem., 2006, 385, 219-224.

9 V. Lednev, S. M. Pershin and A. F. Bunkin, J. Anal. At. Spectrom., 2010, 25, 1745.

10 R. Fantoni, L. Caneve, F. Colao, L. Fornarini, V. Lazic and V. Spizzichino, Spectrochim. Acta, Part B, 2008, 63, 10971108.

11 S. Almaviva, R. Fantoni, L. Caneve, F. Colao, L. Fornarini, A. Santagata and R. Teghil, Spectrochim. Acta, Part B, 2014, 99, 185-192.

12 B. Salle, P. Mauchien, S. Maurice, B. Sallé, P. Mauchien and S. Maurice, Spectrochim. Acta, Part B, 2007, 62, 739-768.

13 P. Rohwetter, J. Yu, G. Mejean, K. Stelmaszczyk, E. Salmon, J. Kasparian, J.-P. Wolf, L. Woste, G. Mejean, K. Stelmaszczyk, E. Salmon, J. Kasparian, J.-P. Wolf and L. Woste, J. Anal. At. Spectrom., 2004, 19, 437-444.

14 J. Ruiz, T. Delgado, L. M. Cabalin and J. J. Laserna, J. Anal. At. Spectrom., 2017, 32, 1119-1128.

15 C. M. Davies, H. H. Telle, D. J. Montgomery and R. E. Corbett, Spectrochim. Acta, Part B, 1995, 50, 1059-1075.

16 D. A. Cremers, J. E. Barefield and A. C. Koskelo, Appl. Spectrosc., 1995, 49, 857-860.

17 A. G. Grigoryants, A. Y. Stavertiy, K. O. Bazaleeva, T. Y. Yudina, N. A. Smirnova, R. S. Tretyakov and A. I. Misyurov, Weld. Int., 2016, 1-6.

18 S. Palanco and J. J. Laserna, J. Anal. At. Spectrom., 2000, 15, 1321-1327.

19 M. A. Khater, Spectrochim. Acta, Part B, 2013, 81, 1-10.

20 C. Lopez-Moreno, K. Amponsah-Manager, B. W. Smith, I. B. Gornushkin, N. Omenetto, S. Palanco, J. J. Laserna and J. D. Winefordner, J. Anal. At. Spectrom., 2005, 20, 552556.

21 G. Cristoforetti, S. Legnaioli, V. Palleschi, A. Salvetti, E. Tognoni, A. P. Benedetti, F. Brioschi and F. Ferrario, J. Anal. At. Spectrom., 2006, 21, 697-702.

22 J. A. Merten, E. Ewusi-Annan, B. W. Smith and N. Omenetto, J. Anal. At. Spectrom., 2014, 29, 571-577.

23 K. Amponsah-Manager, N. Omenetto, B. W. Smith, I. B. Gornushkin and J. D. Winefordner, J. Anal. At. Spectrom., 2005, 20, 544-551.

24 J.-F. Y. Gravel, F. R. Doucet, P. Bouchard and M. Sabsabi, J. Anal. At. Spectrom., 2011, 26, 1354-1361.

25 Q. Zeng, L. Guo, X. Li, C. He, M. Shen, K. Li, J. Duan, X. Zeng and Y. Lu, J. Anal. At. Spectrom., 2015, 30, 403-409.

26 Q. Zeng, L. Guo, X. Li, M. Shen, Y. Zhu, J. Li, X. Yang, K. Li, J. Duan, X. Zeng and Y. Lu, J. Anal. At. Spectrom., 2016, 31, 767-772.

27 V. P. Veiko, V. N. Lednev, S. M. Pershin, A. A. Samokhvalov, E. B. Yakovlev, I. Y. Zhitenev and A. N. Kliushin, Rev. Sci. Instrum., 2016, 87, 063114.

28 I. S. O. Standard, Lasers and laser-related equipment - Test methods for laser beam widths, divergence angles and beam propagation ratios, ISO Standard, 2005, pp. 11142-11146. 
29 K. Bai, S. Yao, J. Lu, J. Zhao, J. Xu and Z. Lu, J. Anal. At. Spectrom., 2016, 31, 2418-2426.

30 T. A. Labutin, S. M. Zaytsev, A. M. Popov and N. B. Zorov, Opt. Express, 2014, 22, 22382-22387.

31 C. Aragón and J. a. Aguilera, Spectrochim. Acta, Part B, 2008, 63, 893-916.

32 S. Freudenstein and J. Cooper, Astron. Astrophys., 1979, 71, 283-288.

33 C. Aragón, J. A. Aguilera and J. Campos, Appl. Spectrosc., 1993, 47, 606-608.
34 A. Kramida, Y. Ralchenko, J. Reader and NIST ASD Team, NIST Atomic Spectra Database (ver. 5.5.1), National Institute of Standards and Technology, Gaithersburg, MD, 2017, https://physics.nist.gov/asd.

35 W. B. Barnett, Spectrochim. Acta, Part B, 1968, 23, 643-664.

36 C. Aragon, J. A. Aguilera and F. Penalba, Appl. Spectrosc., 1999, 53, 1259-1267.

37 V. Sturm, L. Peter and R. Noll, Appl. Spectrosc., 2000, 54, 1275-1278.

38 M. A. Khater, J. T. Costello and E. T. Kennedy, Appl. Spectrosc., 2002, 56, 970-983. 